\title{
Analysis and Research on Causes of Metro Accidents Based on 24 Model
}

\author{
Xiaoxiao TANG ${ }^{1,2}$ a, Chenlei LI ${ }^{1,2}$ b, Lei LI ${ }^{1,2,{ }^{*} c}$,Yanhua WU ${ }^{3}$ \\ ${ }^{1}$ Key Laboratory of Urban Rail Transit Intelligent Operation and Maintenance Technology \& Equipment of Zhejiang Province \\ ${ }^{2}$ College of Engineering Zhejiang Normal University Jinhua, China \\ ${ }^{3}$ Institute of Computing Technologies China Academic of Railway Sciences Corporation Limited Beijing, China
}

\begin{abstract}
In order to strengthen the operational safety of the metro system, a 24 model is proposed for metro accident in this study, which is conducive to scientifically and efficiently carrying out metro accident analysis and targeted preventive measures. Referring to the 24 model, the elements that threaten the safety of metro operations are classified, and then performed in individual-level and organizational-level causal analysis one by one on "Shanghai Metro 9.27 Accident", including unsafe human behavior, unsafe material condition, safety knowledge, safety awareness, safety management system, safety culture. The analysis results indicate that the various cause modules defined by the 24 model accomplish metro accident analysis systematically and accident prevention should start from identifying the unsafe material conditions, controlling the unsafe human behaviors, learning safety knowledge, cultivating safety consciousness, perfecting safety management system and so on.
\end{abstract}

\section{Introduction}

With the continuous advancement of urbanization, the construction of global urban rail transit has been rapidly developed. Relevant statistics figure that by the end of 2020, there are 538 cities in 77 countries have developed urban railway system, mileage has already amounted to 33,346 kilometers. Among them, the quantity in China is achieving at $7978.19 \mathrm{~km}$, which is the top 1 in the world and has priority over other countries [1-2]. Overall, 6 cities in China are among the world's top 10 cities in terms of urban rail transit mileage. It can be seen that the mileage of urban rail transit in China has continued to rise, and China has become the country with the most rapid development of rail transit in the world. However, as the complexity of the metro system and the uncertainty of the external environment increasing, various international and domestic metro safety accidents frequently occur. Until now, there have been metro safety accidents at home and abroad, especially the Shanghai metro rear-end collision (2011) and the Mexico City metro train collision (2020), which have caused great losses to safety of life, property and social stability. In this context, for the purpose of preventing such accidents, it is necessary to take reasonable measures to carry out accident cause research, analyze major metro accidents and locate the cause of the accident, which has realistic significance to prevent metro accidents, reduce accident losses and improve the safety of metro operation. This paper will use the 24 model to analyze subway operation accidents from a new perspective.
At present, many scholars have carried out related theoretical analysis and investigations on the causes of accidents. The accident analysis models involved can be divided into three categories. Initially, simple linear methods, such as Domino Model, are not suitable for analysis of complex systems, since they only have a simple causal relationship between two factors, can hardly handle accidents caused by the mutual influence of multiple factors. After that, complex linear methods, such as the Swiss Cheese Model, believe that accidents are the result of superposition of random events, and cannot accurately represent the nonlinear and dynamic characteristics of the interaction of complex socialtechnical system modules. Lastly, the system theory method considers the function and interaction of the entire system as well as the performance variability or control level of the system, can promote the analysis results more rational. Thus, the system theory method has become a hot topic in the research of safety issues in recent years, such as HFACS, AcciMap, STAMP, FRAM, 24 model.

In recent years, accidents data has demonstrated that the influence of individual and organization plays a vital role in the modern systems. However, the uncertainty of human and organizational factors accentuated the difficulty of accident research, resulting in the domestic research is still in its infancy. The 24 model, as a causal model of system accidents, deems that accident analysis should start with organization level and individual level. According to the relationship between the development of accidents, the process is divided into organizational behavior triggers individual behavior and individual behavior directly leads to behavioral results. 24 model is

\footnotetext{
atangxiaoxiao@zjnu.edu.cn ${ }^{\text {b} E c h o l i @ z j n u . e d u . c n ~}$

c* Lei LI: zjnulilei@163.com
} 
highly operable since it systematically defines the cause modules of accidents and clarifies the methods and steps of accident analysis, which can provide researchers with obvious thoughts of accident analysis, to some extent, avoid the occurrence of different analysis results due to insufficient knowledge of researchers [3]. Until now, the 24 model has been applied in social security, chemical industry, transportation and other fields [4-7], while it's rarely used in the metro field. With reference to previous research, in this study, we establish a classification framework for the cause of metro accidents based on the 24 model, and apply the framework to the cause analysis of the "Shanghai Metro 9.27 Accident" in order to provide some reference recommendations for metro security and at the same time furnish metro operating system with strong theoretical supports.

\section{Causal Analysis Method of Metro Accidents Based on 24 Model}

Fu et al. proposed a behavior-based accident causation 2 model suitable for the cause of modern accidents in 2005, which based on the comprehensive analysis of the accident causation chains and Swiss Cheese Model (SCM). And in the following years, it was improved and perfected to form the final model, which is shown in Figure 1. The model indicates that the cause of accidents can divided into four stages at the individual and organizational levels.

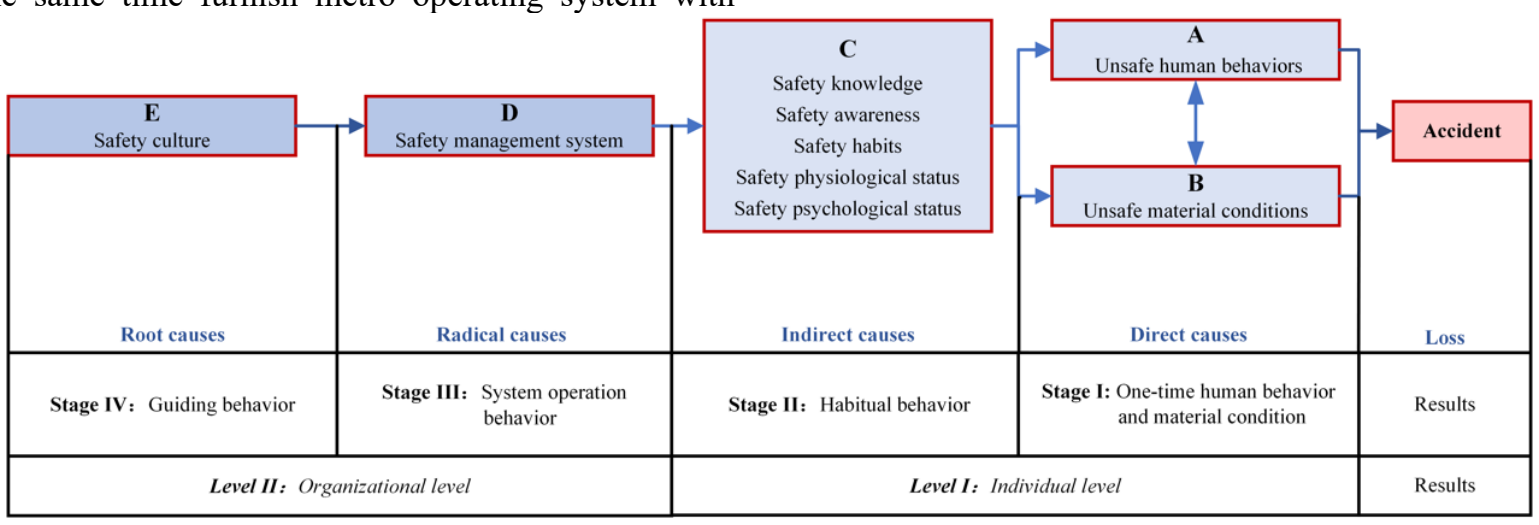

Fig1. 24 Model.

Stage I: One-time human behavior and material condition-Direct causes. 24 model attributes the direct causes of the accident to human unsafe behavior and unsafe condition based on relevant research results which has been widely recognized at home and abroad.

Stage II: Habitual behavior-Indirect causes. On the basis of sufficiently consideration of previous studies, 24 model divides indirect causes into five classifications, namely safety knowledge, safety awareness, safety habits, psychological statue and physiological statue. When one or more of these factors cause problems, it usually gives rise to unsafe behavior, which in turn can bring about unsafe events.

Stage III: System operation behavior-Radical causes. Modern accident causation models usually regard organizational or management factors as the radical causes of accidents. Combined with modern safety management theory, 24 model enriches it into a SMS, which takes charge of the safety production of the enterprise. When the SMS has defects or confusion, it usually brings latent dangers to production.

Stage IV: Guiding behavior-Root causes. Safety culture, namely corporate culture, is deemed as the root causes in the 24 model. Safety culture works through the SMS, it makes specific requirements for behavior standards and corporate environment, guides the actions of the staff, restricts the unsafe behavior, so as to achieve the purpose of preventing accidents.

In the 24 model, human behaviors could be controlled compared to the material conditions. Human behaviors are not only dependent on safety knowledge, safety awareness, safety habits, psychological statue and physiological statue at individual level, but also SMS and safety culture at organizational level. When unsafe behaviors occur, the drawbacks of related factors at the individual and organizational levels could deduced backwards, and finally, remedial and improvement measures could be proposed for the deficiencies found. Hence, the factors affecting metro accidents can be sorted and analyzed on the basis of the 24-model framework as well. The causation analysis model for metro accidents is established as follows:

A: Unsafe human behaviors, such as unsafe behavior of metro staff in daily work or in the process of dealing with emergency events (e.g., train driver furious driving, disoperation or improper operation caused by unfamiliarity in the process of train troubleshooting, maintenance personnel in the failure to check out the potential safety hazards of vehicle, power supply equipment and others or to make a wrong judgment of the existing faults, dispatchers don't report on-site conditions to the dispatch center in a timely manner as required, station attendant fail to play a supervisory role in accordance with the operation plan and other train due to weak safety awareness and other reasons (e.g., passengers are caught by safety doors, fall into orbit, passengers injured by congestion)

B: Unsafe material conditions, such as malfunction of vehicle system (e.g., short out of vehicle circuit, wear or deformed or corrosion fatigue of machine component), vehicle driving risk (e.g., train-to-train collision, train derailment, failure of train master controller, train caught fire), failure of station equipment and facilities ( e.g., breakdown of shielding door inductor, the retaining element of the elevator are damaged causing the escalator schedules), insecurity behavior caused by non-metro staff 
to move backwards), invalidity of power supply system ( e.g., trip-out of power supply catenary, unstable power supply in the tunnel, fire in the substation), malfunction in communication system ( e.g., train signal control equipment is out of order, blackout of block signal machine, signal debugging error), breakdown of other equipment system ( e.g., component deterioration of safety protection system for trains, the defect in station safety system), the extreme weather condition (e.g., failure of mechanical and electrical equipment caused by natural disasters such as snowstorm, rainstorm and earthquake).

C: Habitual behaviors, for example, unsafe behavior of metro staffs indirectly caused by having little knowledge about SMS, studying safety operation rules half-heartedly, lacking of professional knowledge and practical experience (e.g., illegal command, escaped alone at the scene of the accident, failure to convey information or unclear explanation when conveying information), weak sense of safety responsibility or fluke mentality of metro staffs, or failure to fully understand the serious consequences of insecurity behavior (e.g., staffs are unable to wear the personal protective equipment correctly), the metro staffs are in the habit or practice of making the violation operation acquiesced by the commander, which bring about the hidden danger to a certain extent (e.g., form the operation habit of missing partial links), poor emotional and mental state may indirectly give rise to unsafe actions or activate incertitude condition (e.g., operation errors result from Inability to concentrate due to depression, in a poor state of physiological status(e.g., sleepy caused by fatigue, or dysphotia when working in a dark environment).

D: System operation behaviors, the construction of metro SMS is imperfect which mainly include metro SMS, metro safety production policy, metro safety management plan, metro emergency plan and accident handling (e.g., operating rules for metro staff isn't detailed enough, insufficient training on improving staffs' awareness and knowledge of metro safety protection, short of emergency rescue plans after the metro operation accident). In consequence, the establishment and implementation of corresponding management system and procedure documents in metro system should be focused on during the process of analyzing the model.

E: Guiding behaviors, the root of the imperfect metro SMS lies in the absence of security culture in metro companies, that is, the inadequate understanding of the 32 elements of safety culture about metro operation [8]. Culture default could easily give rise to metro staffs from the ideological slack so that the regulations come undone, even if the more perfect metro SMS also cannot be put into effect. For example, metro companies ignore the importance of safety in pursuit of economic interests, or neglect the safety education and training needs of employees in order to reduce safety investment, which aren't conducive to the formation of safety values.

\section{3 "Shanghai Metro 9.27 Accident" Analysis Based on 24 Model}

\subsection{The Background of Accident}

At 13:58 on September 27, 2011, when the cable hole was blocked at Xintiandi Station of Metro Line 10, power supply was lacking, and the signal at Xintiandi Central Station of Metro Line 10 lost power. At 14:00, the 1016 train stopped at a red light at the interval from Yuyuan Station to Laoximen Station. At 14:08, uplinks and downlinks from Jiaotong University Station to Nanjing East Road Station adopt the telephone blocking method, the train run at a limited speed. At 14:35, when the 1005 train was moving towards the Laoximen station, it was found that the 1016 train staying in front, even though the 1005 train immediately took braking measures, at 14:37, rear-end collision occurred between 1005 train and the 1016 train.

\subsection{One-time Human Behaviors and Material Conditions}

1)Unsafe human behaviors. A1: In the process of handling the emergency incident of malfunctioning signal equipment at Xintiandi Station, the metro controller issued a dispatch instruction for the implementation of telephone blocking without confirming the specific locations of other 6 trains in the accident section; A2: The operator on duty at the receiving station did not strictly comply with the train operation diagram and temporary operation plan, illegally agreed with the telephone blocking requirements of the departure station.

2)Unsafe material conditions. B1: There were holes in the cables under UPS cabinets at Xintiandi Station of Metro Line 10; B2: The power supply system was out of order; B3: The communication system was abnormal, display device exhibited that the track section was occupied or malfunctioning, and the automatic monitoring panel of trains depicted in black.

\subsection{Habitual Behaviors}

1)Lack of safety knowledge. C1: The staff didn't master the safety technical characteristics so that they couldn't dig the potential problems affecting metro operation as expected before the accident; C2: When performing their responsibilities for the rail line, dispatchers and station attendants failed to review and monitor the section conditions for they didn't grasp the operation rules of safety production; C3: Staffs on duty were lack of emergency knowledge, and their responsibilities were not clearly demarcated when dealing with emergencies related to signal interruption.

2)Weak safety awareness. C4: The power supply company, Shanghai Metro First Operation Co., Ltd. and the construction personnel lacked awareness of safety responsibility, they didn't assess the risks of construction operations and formulate corresponding preventive measures before carrying out facility maintenance operations, nor did they formulate working plan for the 
train operation period; C5: Dispatchers neglected consequences of their action, during the implementation of the telephone blocking, they failed to confirm that the section from Yuyuan Station to Laoximen Station was free, and agreed to transact the telephone blocking of train 1005.

3)Poor safety habits. C6: Before the accident, Shanghai metro is prone to breakdowns, which have not been paid enough attention by relevant personnel, so that they never consider the root cause from the overall perspective of metro safety, but deal with it cautiously.

4)Poor safety psychology. C7: Before the accident, the metro of line 10 traveled in the wrong direction, but the staff firmly believed the strict automatic protection system would prevent the train from rear-ending.

\subsection{System Operation Behaviors}

1)Unexhaustive rules and regulations. D1: Shanghai Metro First Operation Co., Ltd. failed to implement national and municipal regulations on metro operation safety, and failed to formulate corresponding specific post operating procedures as required, resulting in unclear responsibilities for operators in responding to emergencies; D2: The regulations of telephone blocking were not suitable for metro industry, the standards and specifications of train positioning, tracking and control were not perfect; D3: There was no risk evaluation mechanism, and no regulations on the investigation and treatment of hidden dangers.

2)Inadequate emergency management. D4 The requirements of railway operation management, such as telephone block method and train control system based on wireless communication, were not included in the emergency plan seasonably; D5: Emergency drills were not well-targeted, like the lack of training and drills under abnormal operating conditions, which brought about employees cannot effectively adopt emergency rescue strategies in this circumstance.

3) Weak links exist in team construction and education training. D6: Employees failed to carry out technical operations normally since insufficient job education and training were organized.

4)Lack of supervision and management. D7: Supervision and implementation of metro operation safety production responsibility system was not in place; D8: There was no supervision and inspection of daily scheduling work and implementation of regulations; D9: Safety inspections were not organized regularly to monitor hazard sources.

\subsection{Guiding Behaviors}

The 24 model positions the root cause as the lack of safety concept and safety culture. The degree of realization of safety culture elements reflects the level of construction of safety culture. According to the safety culture elements [8], there were 11 elements of safety culture missing from the accident unit in this case, they respectively were: (1) the safety importance (2) all the accident can be prevented (3) safety create economic benefits (4) safe integration into corporate management (5) safety depends on safety consciousness (6) the main responsibility of security (8) function of safety regulations (9) formation of safety values (13) requirements of safety training (16) the role of the management system (32) emergency ability.

\section{Conclusion}

(1) The 24model creates a reliable theoretical support for the cause analysis of metro accidents. According to the theory and system described by the model, the accident can be analyzed in depth. Follow-up measures to guarantee the safety and stability of the metro system can be formulated from the detection of unsafe material conditions, domination of unsafe human behaviors, enhancement of safety knowledge, cultivation of safety awareness, and improvement of SMS to improve the safety and stability of the metro system. For example, strengthen the management of employees' operational behavior, strengthen safety education and training, improve emergency response capabilities.

(2) Compared with the "Shanghai Metro 9.27 Accident" investigation report, on the one hand, 24 model can generate more comprehensive and detailed analysis results by digging out the deeper accident causes and exploring more methodically organizational factors. On the other hand, the model is more conducive to formulating risk control measures in a targeted manner due to the reason modules have clear boundaries and logical relationships.

\section{Acknowledgment}

This work was supported by the China Academy of Engineering Consulting Research Project (2019-ZD-39). The authors are also appreciate for the assistance from members of the research group in providing suggestions for revision of the paper.

\section{References}

1. China Association of Metros. (2020) Overview of urban rail transit lines in mainland China. https: //www. camet.org.cn/xxfb/7270.

2. Han, B. M., Dai, W., \& Zhang, H. J. (2019) Statistics and analysis of urban rail transit operation in the world. Urban Rapid Rail Transit, 32(1), 9-14.

3. Monferini, A., Konstandinidou, M., Nivolianitou, Z., Weber, S., Kontogiannis, T., Kafka, P., ... \& Demichela, M. (2013) A compound methodology to assess the impact of human and organizational factors impact on the risk level of hazardous industrial plants. Reliability Engineering \& System Safety, 119, 280289.

4. Chen, M., Wu, Y., Wang, K., Guo, H., Ke, W. (2020) An explosion accident analysis of the laboratory in university. Process Safety Progress, 39(4), e12150.

5. Wu, Y., Chen, M., Niu, L., Qiu, P., Zhou, A. (2020) A new safety supervision model for underground coal mines in China. Energy Sources, Part A: Recovery, 
Utilization, and Environmental Effects, 1-15.

6. Huang, W., Shuai, B., Zuo, B., Xu, Y., Antwi, E. (2019)

A systematic railway dangerous goods transportation system risk analysis approach: The 24 model. Journal of Loss Prevention in the Process Industries, 61, 94103.

7. Fu G, Suo X, Wang C X. (2018) Study on the systematic characteristics of 24Model. Systems Engineering - Theory \& Practice, 38(1): 263-272.

8. ZHANG, F. G. H. D. Y., Ji-ye, S. D. (2013) Further discussions on definition of safety culture and its assessment indicators. China Safety Science Journal, 23(04):140-145. 